\title{
On Analytic Properties of the Photon Polarization Function in a Background Magnetic Field
}

\author{
W. F. Kao, Guey-Lin Lin and Jie-Jun Tseng \\ Institute of Physics, National Chiao Tung University, Hsinchu 300, Taiwan
}

(November 2, 2018)

\begin{abstract}
We examine the analytic properties of the photon polarization function in a background magnetic field, using the technique of inverse Mellin transform. The photon polarization function is first expressed as a power series of the photon energy $\omega$ with $\omega<2 m_{e}$. Based upon this energy expansion and the branch cut of the photon polarization function in the complex $\omega$ plane, we compute the absorptive part of the polarization function with the inverse Mellin transform. Our results are valid for arbitrary photon energies and magnetic-field strengths. The applications of our approach are briefly discussed.
\end{abstract}

PACS numbers: 12.20.Ds, 11.55.Fv

The behavior of a charged particle in a background magnetic field is rather well known. The energy of the charged particle is quantized according to the Landau levels. For a non-relativistic electron moving in a uniform magnetic field along the $+z$ direction, its energy levels are given by

$$
E_{n, s_{z}}=\left(n+\frac{1}{2}+s_{z}\right) \omega_{c}+\frac{p_{z}^{2}}{2 m_{e}}
$$

where $\omega_{c} \equiv e B / m_{e}(e>0)$ is the cyclotron frequency of the electron and $s_{z}$ is the electron spin projection along the $+z$ direction. For a relativistic electron (positron), the energy quantization becomes

$$
E_{n, s_{z}}^{2}=m_{e}^{2}+p_{z}^{2}+e B\left(2 n+1+2 s_{z}\right),
$$

with the following correspondent wave function

$$
\psi_{n, p_{y}, p_{z}, s_{z}}^{ \pm}(\vec{r}, t)=\exp \left(-i E_{n} t+i p_{y} y+i p_{z} z\right) \mathbf{F}_{n, p_{z}, s_{z}}^{ \pm}\left(x^{\prime}\right)
$$

where $\mathbf{F}_{n, p_{z}, s_{i n}}^{ \pm}\left(x^{\prime}\right)$ is a four-component spinor with $x^{\prime}=x-p_{y} / e B$. The detailed form for $\mathbf{F}_{n, p_{z}, s_{z}}^{ \pm}$can be found, for example, in [1].

While the wave function of a charged fermion in the background magnetic field is well understood, it is often rather non-trivial to compute a physical process occurring in a background magnetic field, particularly, if there are more than one charged fermions involved in the reaction. A famous example is the pair production process $\gamma \rightarrow e^{+} e^{-}$, which is relevant to the gamma-ray attenuation in the neutron star [2], and was first studied by Toll [3] and Klepikov [4] independently. The approach by Toll and Klepikov is based upon a direct squaring of $\gamma \rightarrow e^{+} e^{-}$matrix elements using exact electron and positron wave functions in a background magnetic field. The most updated calculation using this approach can be found in [5. To understand this problem better, however, one should demonstrate that the pair production width can also be obtained from the absorptive part of photon polarization function in a background magnetic field. In this regard, Tsai and Erber $[6]$ obtained the absorptive part of the one-loop photon polarization function in the asymptotic limit $\omega \gg 2 m_{e}$ and $B \ll B_{c} \equiv m_{e}^{2} / e$. Their result was shown [6] to agree with that of Toll and Klepikov. However, in the aforementioned asymptotic limit, the threshold behavior of the pair-production width is completely absent. Later on Shabad [7] obtained the absorptive part (and the dispersive part as well) of one-loop photon polarization function for a general photon energy and magnetic-field strength. In that work, the threshold behavior of the pair-production width was worked out explicitly. We remark that Refs. [6,7] employed Schwinger's proper-time representation for the electron and positron Green's functions [8] inside the photon polarization functions. To our knowledge, Ref. [7] is the first work which shows that the proper-time representation for the photon polarization function gives equivalent pair-production width to that obtained from squaring the $\gamma \rightarrow e^{+} e^{-}$amplitude directly. Unfortunately, the manipulations of Ref. [7] are rather involved and the substantial details of them were given in some other unpublished preprints [9]. It is not very clear how one can generalize the approach of Ref. [7] to other processes.

In this work, we will provide an alternative derivation of the pair-production width (or equivalently the absorptive part of the photon polarization function) from the proper-time representation of the photon polarization function. We shall also outline the procedure of obtaining the dispersive part of the polarization function, which is relevant to the photon index of refraction. It will be clear that our approach is very straightforward and physically intuitive. Furthermore, it is applicable to many processes occurring in a constant background electromagnetic field. In fact, 
there are growing activities on computing the two-point and three-point current correlation functions in a constant background field, using the string-inspired world-line formalismli. The final results of these calculations are expressed in terms of multiple integrals similar to the proper-time representations. Our approach will be very useful for investigating the analytic properties of these integrals, hence the analytic behaviors of various current correlation functions.

To illustrate our approach, let us begin with the proper-time representation of photon polarization function $\Pi_{\mu \nu}$ in a constant background magnetic field [12]:

$$
\begin{aligned}
\Pi_{\mu \nu}(q) & =-\frac{e^{3} B}{(4 \pi)^{2}} \int_{0}^{\infty} d s \int_{-1}^{+1} d v\left\{e ^ { - i s \phi _ { 0 } } \left[\left(q^{2} g_{\mu \nu}-q_{\mu} q_{\nu}\right) N_{0}\right.\right. \\
& \left.-\left(q_{\|}^{2} g_{\| \mu \nu}-q_{\| \mu} q_{\| \nu}\right) N_{\|}+\left(q_{\perp}^{2} g_{\perp \mu \nu}-q_{\perp \mu} q_{\perp \nu}\right) N_{\perp}\right] \\
& \left.-e^{-i s m_{e}^{2}}\left(1-v^{2}\right)\left(q^{2} g_{\mu \nu}-q_{\mu} q_{\nu}\right)\right\}
\end{aligned}
$$

where $q$ is the photon four-momentum with $q_{\|}^{\mu} \equiv\left(\omega, 0,0, q_{z}\right)$ and $q_{\perp}^{\mu} \equiv\left(0, q_{x}, q_{y}, 0\right)$ for a magnetic field in the $+z$ direction,

$$
\phi_{0}=m_{e}^{2}-\frac{1-v^{2}}{4} q_{\|}^{2}-\frac{\cos (z v)-\cos (z)}{2 z \sin (z)} q_{\perp}^{2}
$$

with $z=e B s$, and

$$
\begin{aligned}
& N_{0}=\frac{\cos (z v)-v \cot (z) \sin (z v)}{\sin (z)}, \\
& N_{\|}=-\cot (z)\left(1-v^{2}+\frac{v \sin (z v)}{\sin (z)}\right)+\frac{\cos (z v)}{\sin (z)}, \\
& N_{\perp}=-\frac{\cos (z v)}{\sin (z)}+\frac{v \cot (z) \sin (z v)}{\sin (z)}+2 \frac{\cos (z v)-\cos (z)}{\sin ^{3}(z)} .
\end{aligned}
$$

The two independent eigenmodes of the above polarization tensor are $\epsilon_{\|}^{\mu}$ and $\epsilon_{\perp}^{\mu}$ which are respectively parallel and perpendicular to the plane spanned by the photon momentum $\mathbf{q}$ and the magnetic field $\mathbf{B}$. They obey the eigenvalue equations $\epsilon_{\|}^{\mu}\left(-q^{2} g_{\mu \nu}+\Pi_{\mu \nu}\right) \epsilon_{\|}^{\nu} \equiv q^{2}+\Pi_{\|}=0$ and $\epsilon_{\perp}^{\mu}\left(-q^{2} g_{\mu \nu}+\Pi_{\mu \nu}\right) \epsilon_{\perp}^{\nu} \equiv q^{2}+\Pi_{\perp}=0$ respectively with $\Pi_{\|, \perp}=\epsilon_{\|, \perp}^{\mu} \Pi_{\mu \nu} \epsilon_{\|, \perp}^{\nu}$. It turns out that $\Pi_{\|}$and $\Pi_{\perp}$ are proportional to $N_{\|}$and $N_{\perp}$ respectively. We shall not discuss the contribution by $N_{0}$ since it does not correspond to an independent eigenmode. The absorptive part of $\Pi_{\|, \perp}$ gives rise to the photon absorption coefficient (pair-production width) via the relation $\kappa_{\|, \perp}=\operatorname{Im} \Pi_{\|, \perp} / \omega$.

To study the analytic properties of Eq. (4), we employ the sum rule developed by us [13]:

$$
\left.\frac{1}{n !}\left(\frac{d^{n}}{d\left(\omega^{2}\right)^{n}} \Pi_{\|, \perp}\right)\right|_{\omega^{2}=0}=\frac{M_{\|, \perp}^{1-2 n}}{\pi} \int_{0}^{1} d y_{\|, \perp} \cdot y_{\|, \perp}^{n-1} \cdot\left(\kappa_{\|, \perp} y_{\|, \perp}^{-1 / 2}\right)
$$

where $M_{\|}$and $M_{\perp}$ are threshold energies for pair productions with $M_{\|}^{2}-q_{z}^{2}=4 m_{e}^{2}$ and $M_{\perp}^{2}-q_{z}^{2}=m_{e}^{2}(1+$ $\left.\sqrt{1+2 B / B_{c}}\right)^{2} ; y_{\|, \perp}=M_{\|, \perp}^{2} / \omega^{2}$. One notes that the absorptive part of $\Pi_{\|, \perp}\left(\omega^{2}\right)$ vanishes for the range $0 \leq \omega^{2} \leq$ $M_{\|, \perp}^{2}$ [14. Therefore one can effectively set the integration range of Eq. (7) as from $y_{\|, \perp}=0$ to $y_{\|, \perp}=\infty$. Now, it is easily seen that the derivatives of $\Pi_{\|, \perp}$ at the zero energy are proportional to the Mellin transform of $\kappa_{\|, \perp} \cdot y_{\|, \perp}^{-1 / 2} \equiv \kappa_{\|, \perp} \cdot \omega / M_{\|, \perp}$. Once the l.h.s. of Eq. (7) is calculated, the absorption coefficients $\kappa_{\|, \perp}$ or $\operatorname{Im} \Pi_{\|, \perp}$ can be determined by the inverse Mellin transform.

In order to compute the derivatives of $\Pi_{\|, \perp}$ at the zero energy, we note that a rotation of the integration contour $s \rightarrow-i s$ is permissible for a photon energy below the pair-production threshold. This rotation turns the oscillating trigonometric functions in $\Pi_{\|, \perp}$ into more manageable hyperbolic functions. In the limit that $B \ll B_{c}$, one can perform an asymptotic expansion in $B / B_{c}$, which gives

$$
\left.\frac{1}{n !}\left(\frac{d^{n}}{d\left(\omega^{2}\right)^{n}} \Pi_{\|, \perp}\right)\right|_{\omega^{2}=0}=\frac{2 \alpha_{e} m_{e}^{2}}{\pi}\left(\frac{B^{2} \sin ^{2} \theta}{3 B_{c}^{2} m_{e}^{2}}\right)^{n} \frac{\Gamma(3 n-1) \Gamma^{2}(2 n)}{\Gamma(n) \Gamma(4 n)}\left(\frac{6 n+1,3 n+1}{4 n+1}\right)+\cdots,
$$

\footnotetext{
${ }^{1}$ For an overview on recent developments, see [10,11].
} 
where $\theta$ is the angle between directions of the photon momentum and the magnetic field, and the neglected terms are higher order in $B / B_{c}$. Applying the inverse Mellin transform, we obtain absorption coefficients $\kappa_{\|, \perp}$ in agreement with previous results by Tsai and Erber [6]. Such results are however not satisfactory. As one can easily see, apart from the trivial mass factor $m_{e}^{2-2 n}$, the first term on the R.H.S. of Eq. (8) grows to infinity as $n$ increases, no matter how small the ratio $B / B_{c}$ is. This implies that the disregarded higher-order terms are in fact non-negligible for a sufficiently large $n$. As a result, the large moments of $\kappa_{\|, \perp}$ are not accurately determined by the first term of the above asymptotic expansion. Hence the threshold behaviors of $\kappa_{\|, \perp}$ are not seen after applying the inverse Mellin transform. To generate correct threshold behaviors for absorption coefficients, we shall not expand in the magnetic-field strength even if $B \ll B_{c}$. We will obtain photon absorption coefficients valid for arbitrary photon energies and magnetic-field strengths.

It is simpler to analyze $\Pi_{\|, \perp}$ in the special case that $q_{z}=0$, i.e., $\mathbf{q} \cdot \mathbf{B}=0$. Without losing the generality, we may further choose $q^{\mu}=\left(\omega, q_{x}, 0,0\right)$. It is easy to show that $\Pi_{\|}=-\left(\alpha_{e} \omega^{2} / 4 \pi\right) \bar{\Pi}_{\|}$and $\Pi_{\perp}=-\left(\alpha_{e} q_{x}^{2} / 4 \pi\right) \bar{\Pi}_{\perp}$ with

$$
\begin{aligned}
& \bar{\Pi}_{\|}\left(\omega^{2}, q_{x}^{2}\right)=\int_{0}^{\infty} d z \int_{-1}^{+1} d v \exp \left[-i s \phi_{0}\right] N_{\|}, \\
& \bar{\Pi}_{\perp}\left(\omega^{2}, q_{x}^{2}\right)=\int_{0}^{\infty} d z \int_{-1}^{+1} d v \exp \left[-i s \phi_{0}\right] N_{\perp} .
\end{aligned}
$$

Clearly, for the general case that $q^{\mu}=\left(\omega, q_{x}, 0, q_{z}\right)$ with $q_{z}^{2}=q_{x}^{2} \cot ^{2} \theta$, the photon polarization functions, denoted as $\hat{\Pi}_{\|, \perp}\left(\omega^{2}, q_{x}^{2}, \theta\right)$, are given by $\hat{\Pi}_{\|}\left(\omega^{2}, q_{x}^{2}, \theta\right)=-\left(\alpha_{e} \omega^{2} \sin ^{2} \theta / 4 \pi\right) \bar{\Pi}_{\|}\left(q_{\|}^{2}, q_{x}^{2}\right)$, with $q_{\|}^{2} \equiv \omega^{2}-q_{z}^{2}=\omega^{2}-q_{x}^{2} \cot ^{2} \theta$; and $\hat{\Pi}_{\perp}\left(\omega^{2}, q_{x}^{2}, \theta\right)=-\left(\alpha_{e} q_{x}^{2} / 4 \pi\right) \bar{\Pi}_{\perp}\left(q_{\|}^{2}, q_{x}^{2}\right)$. Let us now return to the special case. Rotating the integration contour $s \rightarrow-i s$ and performing the energy expansion in $\omega$, we arrive at

$$
\Pi_{\|}\left(\omega^{2}, q_{x}^{2}\right)=-\frac{\alpha_{e} \omega^{2}}{4 \pi}\left(A\left(\alpha_{a}, \beta_{a}\right)+B\left(\alpha_{b}, \beta_{b}\right)+B\left(\alpha_{b}^{\prime}, \beta_{b}^{\prime}\right)+C\left(\alpha_{c}, \beta_{c}\right)+C\left(\alpha_{c}^{\prime}, \beta_{c}^{\prime}\right)\right),
$$

and

$$
\Pi_{\perp}\left(\omega^{2}, q_{x}^{2}\right)=\frac{\alpha_{e} q_{x}^{2}}{4 \pi}\left(A\left(\alpha_{a}, \beta_{a}\right)+C\left(\alpha_{c}, \beta_{c}\right)+C\left(\alpha_{c}^{\prime}, \beta_{c}^{\prime}\right)+D\left(\alpha_{d}, \beta_{d}\right)\right),
$$

where the functions $A, B, C$ and $D$ are defined as

$$
\begin{aligned}
& A(\alpha, \beta)=\sum K_{l m p p^{\prime}}^{A}\left(q^{\prime 2}\right)\left(\frac{1}{B^{\prime}}\right)^{n} \frac{\left(\omega^{\prime 2}\right)^{n-l}}{(n-l) !} \int_{0}^{\infty} d z z^{n-l} \exp [-z \beta] \int_{0}^{1} d v\left(1-v^{2}\right)^{n-l}(\exp [\alpha z v]+\exp [-\alpha z v]) \\
& B(\alpha, \beta)=-\sum K_{l m p p^{\prime}}^{B}\left(q^{\prime 2}\right)\left(\frac{1}{B^{\prime}}\right)^{n} \frac{\left(\omega^{\prime 2}\right)^{n-l}}{(n-l) !} \int_{0}^{\infty} d z z^{n-l} \exp [-z \beta] \int_{0}^{1} d v\left(1-v^{2}\right)^{n-l+1}(\exp [\alpha z v]+\exp [-\alpha z v]) \\
& C(\alpha, \beta)=-\sum K_{l m p p^{\prime}}^{C}\left(q^{\prime 2}\right)\left(\frac{1}{B^{\prime}}\right)^{n} \frac{\left(\omega^{\prime 2}\right)^{n-l}}{(n-l) !} \int_{0}^{\infty} d z z^{n-l} \exp [-z \beta] \int_{0}^{1} d v v\left(1-v^{2}\right)^{n-l}(\exp [\alpha z v]-\exp [-\alpha z v]) \\
& D(\alpha, \beta)=-\sum K_{l m p p^{\prime}}^{D}\left(q^{\prime 2}\right)\left(\frac{1}{B^{\prime}}\right)^{n} \frac{\left(\omega^{\prime 2}\right)^{n-l}}{(n-l) !} \int_{0}^{\infty} d z z^{n-l} \exp [-z \beta] \int_{0}^{1} d v\left(1-v^{2}\right)^{n-l}(\exp [\alpha z v]+\exp [-\alpha z v])
\end{aligned}
$$

with $\sum \equiv \sum_{n=0}^{\infty} \sum_{l=0}^{n} \sum_{m=0}^{\infty} \sum_{p^{\prime}, p=0}^{l}, \omega^{\prime 2} \equiv \omega^{2} / 4 m_{e}^{2}, B^{\prime} \equiv B / B_{c}, q^{\prime 2} \equiv q_{x}^{2} / 4 m_{e}^{2}$,

$$
\begin{aligned}
& K_{l m p p^{\prime}}^{A}\left(q^{\prime 2}\right)=\frac{2(-1)^{l+p+p^{\prime}}\left(2 q^{\prime 2}\right)^{l} \Gamma(l+m+1)}{(l-p) ! p !\left(l-p^{\prime}\right) ! p^{\prime} ! \Gamma(m+1)}, \quad K_{l m p p^{\prime}}^{B}\left(q^{\prime 2}\right)=\frac{1}{2} K_{l m p p^{\prime}}^{A}\left(q^{\prime 2}\right), \\
& K_{l m p p^{\prime}}^{C}\left(q^{\prime 2}\right)=K_{l m p p^{\prime}}^{A}\left(q^{\prime 2}\right)\left(\frac{l+m+1}{l+1}\right), \quad K_{l m p p^{\prime}}^{D}\left(q^{\prime 2}\right)=\frac{8(-1)^{l+p+p^{\prime}}\left(2 q^{\prime 2}\right)^{l} \Gamma(l+m+3)(l+1)}{(l+1-p) ! p !\left(l+1-p^{\prime}\right) ! p^{\prime} !(l+2) \Gamma(m+1)} .
\end{aligned}
$$

The actual arguments in the functions $A, B, C$, and $D$ are given by $\alpha_{a}=\alpha_{c}=\alpha_{c}^{\prime}=p^{\prime}-p+1, \alpha_{b}=\alpha_{b}^{\prime}=\alpha_{d}=p^{\prime}-p$, $\beta_{a}=p+p^{\prime}+2 m+1+1 / B^{\prime}, \beta_{b}=\beta_{a}-1, \beta_{b}^{\prime}=\beta_{a}+1, \beta_{c}=\beta_{a}, \beta_{c}^{\prime}=\beta_{a}+2$, and $\beta_{d}=\beta_{a}+1$. Note that the various indices

\footnotetext{
${ }^{2}$ For convenience, we shall suppress the subscripts of $\alpha$ and $\beta$ except in some special cases.
} 
in the summation arise as follows: the index $n$ comes from the photon-energy expansion, $l$ arises from the binomial expansion of $\left(\left(1-v^{2}\right) \omega^{\prime 2}+2(\cosh (z v)-\cosh (z)) q^{\prime 2} / z \sinh (z)\right)^{n}, p$ and $p^{\prime}$ arises from writing $(\cosh (z v)-\cosh (z))^{l}$ as a sum of exponential functions, and $m$ is due to expansions such as $\sinh ^{-l} z=2^{l} \exp [-l z] \sum_{m=0}^{\infty} C_{m}^{l+m-1} \exp [-2 m z]$. It may appear at a first glance that our expansion on $\sinh ^{-l} z$ and other similar terms are not convergent at $z=0$. For $z \in(0, \epsilon)$, one should expand $\sinh ^{-l} z$ in powers of $z$ rather than in powers of $\exp (-2 z)$. Fortunately, as $\epsilon \rightarrow 0$, the result obtained with a careful treatment of $\sinh ^{-l} z$ does reduce to the one with $\sinh ^{-l} z$ expanded naively.

The computations of $A, B, C$, and $D$ are rather similar in nature. For illustrations, we will go through the details of computing $A(\alpha, \beta)$ and its relevant quantities. First of all, the integration over $v$ can be carried out to give

$$
A(\alpha, \beta)=\sum \sqrt{\pi} K_{l m p p^{\prime}}^{A}\left(q^{\prime 2}\right)\left(\frac{1}{B^{\prime}}\right)^{n} \frac{\left(\omega^{\prime 2}\right)^{n-l}}{\Gamma\left(n-l+\frac{3}{2}\right)} \int_{0}^{\infty} d z z^{n-l} \exp [-z \beta]_{0} F_{1}\left(n-l+\frac{3}{2} ; \frac{\alpha^{2}}{4} z^{2}\right)
$$

where ${ }_{0} F_{1}$ is the generalized hypergeometric function. We then perform the $z$ integration which gives

$$
A(\alpha, \beta)=\sum K_{l m p p^{\prime}}^{A}\left(q^{\prime 2}\right) \sqrt{\pi}\left(\frac{1}{B^{\prime}}\right)^{n} \frac{\left(\omega^{\prime 2}\right)^{n-l}}{(\beta)^{n-l+1}} \frac{\Gamma(n-l+1)}{\Gamma\left(n-l+\frac{3}{2}\right)}{ }_{2} F_{1}\left(\frac{n-l+1}{2}, \frac{n-l+2}{2} ; n-l+\frac{3}{2} ; \frac{\alpha^{2}}{\beta^{2}}\right) .
$$

For later conveniences, it is desirable to disentangle the indices $n$ and $l$. We do this by replacing $n-l+1$ with $n$ and replacing the summation $\sum$ with the summation $\sum^{\prime} \equiv \sum_{n=1}^{\infty} \sum_{l=0}^{\infty} \sum_{m=0}^{\infty} \sum_{p^{\prime}, p=0}^{l}$. This then gives rise to

$$
\Pi_{\|, A(\alpha, \beta)}=-\frac{\alpha_{e} m_{e}^{2}}{\sqrt{\pi}} \sum^{\prime} K_{l m p p^{\prime}}^{A}\left(q^{\prime 2}\right)\left(B^{\prime}\right)^{1-l}\left(\frac{{\omega^{\prime}}^{2}}{\beta B^{\prime}}\right)^{n} \frac{\Gamma(n)}{\Gamma\left(n+\frac{1}{2}\right)}{ }_{2} F_{1}\left(\frac{n}{2}, \frac{n+1}{2} ; n+\frac{1}{2} ; \frac{\alpha^{2}}{\beta^{2}}\right) .
$$

It is straightforward to compute the absorption coefficient by the inverse Mellin transform. We essentially invert the sum rule given by Eq. (7). However, we should remark that Eq. (7) is derived under the assumption $q^{2}=0$ with the angle between $\mathbf{q}$ and $\mathbf{B}$ kept general. Using this assumption, one has $q_{\|}^{2}=\omega^{2} \sin ^{2} \theta$ with $\theta$ being the angle between $\mathbf{q}$ and B. It is then clear that there is no essential difference for analyzing the branch cut of $\Pi_{\|, \perp}$ either in the complex $q_{\|}^{2}$-plane or in the complex $\omega^{2}$-plane. This is the reason we have chosen $\omega^{2}$ as the variable to set up the sum rule in Eq. (7). However, in the current case, $q^{2}$ is completely general. Hence it is more appropriate to study the analytic structures of $\Pi_{\|, \perp}$ in the complex $q_{\|}^{2}$-plane. We generalize Eq. (7) into

$$
\left.\frac{1}{n !}\left(\frac{d^{n}}{d\left(q_{\|}^{2}\right)^{n}} \hat{\Pi}_{\|, \perp}\right)\right|_{q_{\|}^{2}=0}=\frac{m_{\|, \perp}^{-2 n}}{\pi} \int_{0}^{\infty} d u_{\|, \perp} \cdot u_{\|, \perp}^{n-1} \cdot\left(\omega \hat{\kappa}_{\|, \perp}\right)
$$

where $m_{\|}^{2}=4 m_{e}^{2}, m_{\perp}^{2}=m_{e}^{2}\left(1+\sqrt{1+2 B^{\prime}}\right)^{2}$, and $u_{\|, \perp}=m_{\|, \perp}^{2} / q_{\|}^{2}$. Here we use the notations $\hat{\Pi}_{\|, \perp}$ and $\hat{\kappa}_{\|, \perp}$ to emphasize that the above sum rule holds for a general angle between $\mathbf{q}$ and $\mathbf{B}$. We also note that the differentiations with respect to $q_{\|}^{2}$ are understood as acting on $\bar{\Pi}_{\|}\left(q_{\|}^{2}, q_{x}^{2}\right)$ and $\bar{\Pi}_{\perp}\left(q_{\|}^{2}, q_{x}^{2}\right)$ containing inside $\hat{\Pi}_{\|}$and $\hat{\Pi}_{\perp}$ respectively. Taking the limit $\mathbf{q} \cdot \mathbf{B}=0$ in the above sum rule, we can calculate the absorption coefficients $\kappa_{\|, \perp}$ through the following inverse Mellin transform:

$$
\kappa_{\|, \perp}\left(\omega^{2}, q_{x}^{2}, B\right)=\frac{1}{2 i \omega} \int_{C} d s F_{\|, \perp}\left(s, q_{x}^{2}, B\right)(\bar{\omega})^{2 s},
$$

where $\left.F_{\|, \perp}\left(n, q_{x}^{2}, B\right) \equiv \frac{1}{n !} \frac{d^{n}}{d\left(\bar{\omega}^{2}\right)^{n}} \Pi_{\|, \perp}\right|_{\bar{\omega}^{2}=0}$ with $\bar{\omega}=\omega^{\prime}$ for $\|$ polarization and $\bar{\omega}=2 \omega^{\prime} /\left(1+\sqrt{1+2 B^{\prime}}\right)$ for $\perp$ polarization. The integral transform can be easily performed with the formula

$$
\frac{1}{2 \pi i} \int_{C} d s x^{-s} \frac{\Gamma(s)}{\Gamma\left(s+\frac{1}{2}\right)}{ }_{2} F_{1}\left(\frac{s}{2}, \frac{s+1}{2} ; s+\frac{1}{2} ; z^{2}\right)=\frac{1}{\sqrt{\pi}} \frac{\Theta\left(1-x+\frac{x^{2} z^{2}}{4}\right)}{\sqrt{1-x+\frac{x^{2} z^{2}}{4}}}
$$

One can derive this formula using the integral representation of hypergeometric function ${ }_{2} F_{1}(a, b ; c ; z)=$ $[\Gamma(c) /(\Gamma(b) \Gamma(c-b))] \int_{0}^{1} d t t^{b-1}(1-t)^{c-b-1}(1-t z)^{-a}$ and the fact that $\frac{1}{2 \pi i} \int_{C} d s x^{-s} u^{s-1}=\delta(x-u)$. Therefore, we arrive at

$$
\kappa_{\|, A(\alpha, \beta)}=-\frac{\alpha_{e} m_{e} B^{\prime}}{2 \omega^{\prime}} \sum^{\prime \prime} \frac{K_{l m p p^{\prime}}^{A}\left(q^{\prime 2}\right) \Theta\left(\left(1-\frac{\beta B^{\prime}}{\omega^{\prime 2}}\right)+\left(\frac{\alpha B^{\prime}}{2 \omega^{\prime 2}}\right)^{2}\right)}{B^{\prime l} \sqrt{\left(1-\frac{\beta B^{\prime}}{\omega^{\prime 2}}\right)+\left(\frac{\alpha B^{\prime}}{2 \omega^{\prime 2}}\right)^{2}}}
$$


where $\sum^{\prime \prime} \equiv \sum_{l=0}^{\infty} \sum_{m=0}^{\infty} \sum_{p^{\prime}, p=0}^{l}$. To understand the structure of $\kappa_{\|, A}$, we rewrite the denominator of the above equation as

$$
\sqrt{\left(1-\frac{\beta B^{\prime}}{\omega^{\prime 2}}\right)+\left(\frac{\alpha B^{\prime}}{2 \omega^{\prime 2}}\right)^{2}}=\frac{1}{\omega^{\prime}} \sqrt{\omega^{\prime 2}-1-\left(l_{1}+l_{2}\right) B^{\prime}+\left(l_{1}-l_{2}\right)^{2} \frac{B^{\prime 2}}{4 \omega^{\prime 2}}}
$$

with $(\beta+\alpha) B^{\prime}=1+2 l_{1} B^{\prime}$, and $(\beta-\alpha) B^{\prime}=1+2 l_{2} B^{\prime}$. By a simple kinematic analysis, one can show that the R.H.S. of Eq. (24) is just $\left|p_{1, z}\right| / m_{e} \omega^{\prime} \equiv\left|p_{2, z}\right| / m_{e} \omega^{\prime}$ where $p_{1, z}$ and $p_{2, z}$ are the $z$-direction momenta of $e^{-}$and $e^{+}$ respectively; while $l_{1}$ and $l_{2}$ are the Landau levels occupied by $e^{-}$and $e^{+}$. The pair-production threshold corresponds to $p_{1, z} \equiv p_{2, z}=0$. This threshold behavior is seen explicitly from the step function in the expression for $\kappa_{\|, A}$. It is worthwhile to point out that the variables $\alpha$ 's and $\beta$ 's, appearing first in Eqs. (10) and (11), do have physical meanings. Taking $A(\alpha, \beta)$ as an example, different values of $\alpha$ and $\beta$ imply a different radius of convergence for the infinite series in $\omega^{\prime}$ (see Eq. (17)). The absorptive part of $A(\alpha, \beta)$ emerges once the scaled photon energy, $\omega^{\prime}$, becomes greater than the radius of convergence. This is reflected by the inverse Mellin transform given by Eq. (22).

It is desirable to further simplify Eq. (23). We first perform the summation over the index $l$ in $K_{l m p p^{\prime}}^{A}$ :

$$
\sum_{l=0}^{\infty} \sum_{p, p^{\prime}=0}^{l} \frac{x^{l} \Gamma(l+m+1)}{(l-p) !\left(l-p^{\prime}\right) !}=\sum_{p, p^{\prime}=0}^{\infty} x^{\bar{p}} e^{x} \Gamma(\hat{p}+m+1) L_{\hat{p}+m}^{\bar{p}-\hat{p}}(-x),
$$

where $x=-2 q^{\prime 2} / B^{\prime} \equiv-q_{x}^{2} /(2 e B), \bar{p}=\max \left\{p, p^{\prime}\right\}, \hat{p}=\min \left\{p, p^{\prime}\right\}$, and $L_{\hat{p}+m}^{\bar{p}-\hat{p}}$ is the Laguerre polynomial. To derive this equation, we have used the relations ${ }_{1} F_{1}(\alpha ; \beta ; x)=e^{x}{ }_{1} F_{1}(\beta-\alpha ; \beta ;-x)$ and $L_{n}^{\alpha}(x)={ }_{1} F_{1}(-n ; \alpha+1 ; x) \times C_{n}^{n+\alpha}$. At this juncture, the summation $\sum^{\prime \prime}$ in Eq. (23) has already turned into $\sum_{p, p^{\prime}=0}^{\infty} \sum_{m=0}^{\infty}$. To proceed, we recall that the actual values for $\alpha$ and $\beta$ in this case are $\alpha_{a}=p^{\prime}-p+1$ and $\beta_{a}=p+p^{\prime}+2 m+1+1 / B^{\prime}$, which correspond to the Landau levels $l_{1}=p^{\prime}+m+1$ and $l_{2}=p+m$. Therefore the above summation is equivalent to $\sum_{l_{1}=1}^{\infty} \sum_{l_{2}=0}^{\infty} \sum_{m=0}^{\hat{l}}$ with $\hat{l}=\min \left\{l_{1}-1, l_{2}\right\}$. By collecting the relevant terms in $\kappa_{\|, A\left(\alpha_{a}, \beta_{a}\right)}$, we can further sum over the index $m$ :

$$
\sum_{m=0}^{\hat{l}} x^{-m} \frac{1}{\Gamma(\bar{l}-m+1)} \frac{1}{\Gamma(\hat{l}-m+1)} \frac{1}{\Gamma(m+1)}=\frac{x^{-\hat{l}}}{\Gamma(\bar{l}+1)} L_{\hat{l}}^{\bar{l}-\hat{l}}(-x),
$$

where $\bar{l}=\max \left\{l_{1}-1, l_{2}\right\}$. With the above summations over $l$ and $m$, we arrive at

$$
\kappa_{\|, A}=\frac{\alpha_{e} m_{e} B^{\prime}}{\omega^{\prime}} \sum_{l_{1}=1}^{\infty} \sum_{l_{2}=0}^{\infty} \frac{\mathbf{T}_{l_{1} l_{2}}^{A}(x) \Theta\left(\left(1-\frac{\beta B^{\prime}}{\omega^{\prime 2}}\right)+\left(\frac{\alpha B^{\prime}}{2 \omega^{\prime 2}}\right)^{2}\right)}{\sqrt{\left(1-\frac{\beta B^{\prime}}{\omega^{\prime 2}}\right)+\left(\frac{\alpha B^{\prime}}{2 \omega^{\prime 2}}\right)^{2}}},
$$

with

$$
\mathbf{T}_{l_{1} l_{2}}^{A}(x)=(-1)^{1+r_{A}} x^{r_{A}} e^{x} \frac{\Gamma\left(\lambda_{A}+1\right)}{\Gamma\left(\lambda_{A}+r_{A}+1\right)} L_{\lambda_{A}}^{r_{A}}(-x) L_{\lambda_{A}}^{r_{A}}(-x),
$$

where $r_{A} \equiv \bar{l}-\hat{l}=\left|l_{1}-l_{2}-1\right|$, and $\lambda_{A} \equiv \hat{l}=\left(l_{1}+l_{2}-\left|l_{1}-l_{2}-1\right|-1\right) / 2$. The type $B$ contributions to $\kappa_{\|}$arise from $B\left(\alpha_{b}, \beta_{b}\right)$ and $B\left(\alpha_{b}^{\prime}, \beta_{b}^{\prime}\right)$, We write down the results without repeating the details:

$$
\kappa_{\|, B}=\frac{\alpha_{e} m_{e} B^{\prime 2}}{2 \omega^{\prime 3}} \sum_{l_{1}=0}^{\infty} \sum_{l_{2}=0}^{\infty} \frac{\mathbf{T}_{l_{1} l_{2}}^{B}(x)\left(\beta-\frac{\alpha^{2} B^{\prime}}{2 \omega^{\prime 2}}\right) \Theta\left(\left(1-\frac{\beta B^{\prime}}{\omega^{\prime 2}}\right)+\left(\frac{\alpha B^{\prime}}{2 \omega^{\prime 2}}\right)^{2}\right)}{\sqrt{\left(1-\frac{\beta B^{\prime}}{\omega^{\prime 2}}\right)+\left(\frac{\alpha B^{\prime}}{2 \omega^{\prime 2}}\right)^{2}}},
$$

where

$$
\mathbf{T}_{l_{1} l_{2}}^{B}(x)=(-1)^{r_{B}} x^{r_{B}} e^{x} \frac{\Gamma\left(\lambda_{B}+1\right)}{\Gamma\left(\lambda_{B}+r_{B}+1\right)} L_{\lambda_{B}}^{r_{B}}(-x) L_{\lambda_{B}}^{r_{B}}(-x)+\left(\lambda_{B} \rightarrow \lambda_{B}-1\right),
$$

\footnotetext{
${ }^{3}$ The absorption coefficient is not written in a symmetrized form for saving the space. Nevertheless, the symmetrization with respect to $l_{1}$ and $l_{2}$ can be easily done as one wishes.
} 
with $r_{B}=\left|l_{1}-l_{2}\right|, \lambda_{B}=\left(l_{1}+l_{2}-\left|l_{1}-l_{2}\right|\right) / 2$. We note that the contribution by $B\left(\alpha_{b}^{\prime}, \beta_{b}^{\prime}\right)$ is incorporated by the replacement $\lambda_{B} \rightarrow \lambda_{B}-1$ in the above equation. Finally, the type $C$ contributions are given by

$$
\kappa_{\|, C}=\frac{\alpha_{e} m_{e} B^{\prime 2}}{2 \omega^{\prime 3}} \sum_{l_{1}=1}^{\infty} \sum_{l_{2}=0}^{\infty} \frac{\mathbf{T}_{l_{1} l_{2}}^{C}(x) \Theta\left(\left(1-\frac{\beta B^{\prime}}{\omega^{\prime 2}}\right)+\left(\frac{\alpha B^{\prime}}{2 \omega^{\prime 2}}\right)^{2}\right)}{\sqrt{\left(1-\frac{\beta B^{\prime}}{\omega^{\prime 2}}\right)+\left(\frac{\alpha B^{\prime}}{2 \omega^{\prime 2}}\right)^{2}}},
$$

where

$$
\mathbf{T}_{l_{1} l_{2}}^{C}(x)=(-1)^{r_{C}} x^{r_{C}} e^{x}\left(l_{1}-l_{2}\right) \frac{\Gamma\left(\lambda_{C}+2\right)}{\Gamma\left(\lambda_{C}+r_{C}+1\right)}\left(L_{\lambda_{C}}^{r_{C}}(-x) L_{\lambda_{C}}^{r_{C}}(-x)-L_{\lambda_{C}+1}^{r_{C}-1}(-x) L_{\lambda_{C}-1}^{r_{C}+1}(-x)\right)+\left(\lambda_{C} \rightarrow \lambda_{C}-1\right),
$$

with $r_{C}=r_{A}$ and $\lambda_{C}=\lambda_{A}$. We like to emphasize that, in $\kappa_{\|}$, the relation between $\omega^{\prime 2}$ and $q^{\prime 2}$ is still kept general. It is the dispersive part of $\Pi_{\|}$that determines the relation between $\omega^{\prime 2}$ and $q^{\prime 2}$. The dispersive part can be calculated using the Kramers-Kronig relation:

$$
\operatorname{Re} \bar{\Pi}_{\|}\left(\omega^{\prime 2}, q^{\prime 2}\right)=\frac{P}{\pi} \int_{1}^{\infty} d t \frac{\operatorname{Im} \bar{\Pi}_{\|}\left(t, q^{2}\right)}{t-\omega^{\prime 2}},
$$

where $P$ stands for evaluating the principle part of the integral, while the dimensionless function $\bar{\Pi}_{\|}\left(\omega^{\prime 2}, q^{\prime 2}\right)$ is related to $\Pi_{\|}\left(\omega^{2}, q_{x}^{2}\right)$ by $\Pi_{\|}\left(\omega^{2}, q_{x}^{2}\right)=-\left(\alpha_{e} \omega^{2} / 4 \pi\right) \bar{\Pi}_{\|}\left(\omega^{\prime 2}, q^{\prime 2}\right)$, as has been shown right above Eq. (9). It is well known that $\operatorname{Re} \Pi_{\|}$is relevant to the photon refractive index $n_{\|}\left(\omega^{\prime}\right)$ through the equation $q^{2}+\operatorname{Re} \Pi_{\|}=0$. Hence

$$
n_{\|}\left(\omega^{\prime}\right)=1+\frac{P}{4 m_{e} \pi} \int_{1}^{\infty} d t \frac{\kappa_{\|}(t)}{\sqrt{t}\left(t-\omega^{\prime 2}\right)}
$$

For illustrations, we evaluate $n_{\|}$for a below threshold energy $\omega^{\prime}<1$ and a super-critical magnetic field $B \gg B_{c}$. To the leading order in $B_{c} / B$, it suffices to include the lowest Landau-level contribution on the R.H.S. of the above equation. Such a contribution is contained in $\kappa_{\|, B}$, i.e.,

$$
\kappa_{\|, B}^{l_{1}=l_{2}=0}=\frac{\alpha_{e} m_{e} B^{\prime}}{2 \omega^{\prime 2} \sqrt{\omega^{\prime 2}-1}} \exp \left(-\frac{2 q^{\prime 2}}{B^{\prime}}\right),
$$

Combining Eqs. (34) and (35), we find

$$
n_{\|}\left(\omega^{\prime}\right)=1+\frac{\alpha_{e} B^{\prime}}{4 \pi \omega^{\prime 2}}\left(\frac{1}{\sqrt{\omega^{\prime 2}\left(1-\omega^{\prime 2}\right)}} \arctan \sqrt{\frac{\omega^{\prime 2}}{1-\omega^{\prime 2}}}-1\right),
$$

which agrees with the previous result [7].

We now turn our attentions to the absorption coefficient $\kappa_{\perp}$. It is useful to rewrite Eq. (21) as

$$
\kappa_{\perp}\left(\omega, q_{x}^{2}, B\right)=\frac{1}{2 i \omega} \int_{C} d s \tilde{F}_{\perp}\left(s, q_{x}^{2}, B\right)\left(\omega^{\prime}\right)^{2 s}
$$

where $\left.\tilde{F}_{\perp}\left(n, q_{x}^{2}, B\right) \equiv \frac{1}{n !} \frac{d^{n}}{d\left(\omega^{\prime 2}\right)^{n}} \Pi_{\perp}\right|_{\omega^{\prime 2}=0}$. With $\kappa_{\perp}$ written in this form, we can easily compute $\kappa_{\perp}$ using the results from $\kappa_{\|}$. Comparing Eqs. (10) and (11), we can easily show that $\kappa_{\perp, A}=-\left(q^{\prime 2} / \omega^{\prime 2}\right) \kappa_{\|, A}$, and $\kappa_{\perp, C}=-\left(q^{\prime 2} / \omega^{\prime 2}\right) \kappa_{\|, C}$. Finally, it is slightly involved to compute the type $D$ contribution. We obtain

$$
\kappa_{\perp, D}=\frac{\alpha_{e} m_{e} B^{\prime}}{2 \omega^{\prime}}\left(\frac{q^{\prime 2}}{\omega^{\prime 2}}\right) \sum_{l_{1}=1}^{\infty} \sum_{l_{2}=1}^{\infty} \frac{\mathbf{T}_{l_{1} l_{2}}^{D}(x) \Theta\left(\left(1-\frac{\beta B^{\prime}}{\omega^{\prime 2}}\right)+\left(\frac{\alpha B^{\prime}}{2 \omega^{\prime 2}}\right)^{2}\right)}{\sqrt{\left(1-\frac{\beta B^{\prime}}{\omega^{\prime 2}}\right)+\left(\frac{\alpha B^{\prime}}{2 \omega^{\prime 2}}\right)^{2}}},
$$

with

$$
\mathbf{T}_{l_{1} l_{2}}^{D}(x)=8(-1)^{1+r_{D}} x^{r_{D}-1} e^{x} \frac{\Gamma\left(\lambda_{D}+1\right)}{\Gamma\left(\lambda_{D}+r_{D}\right)} L_{\lambda_{D}-1}^{r_{D}+1}(-x) L_{\lambda_{D}}^{r_{D}-1}(-x),
$$


with $r_{D}=r_{B}$ and $\lambda_{D}=\lambda_{B}$. Similar to the case of $\|$ polarization, the dispersive part of $\Pi_{\perp}$ can also be calculated using the Kramers-Kronig relation. Since the technique is identical, we will not dwell upon this issue again.

We have compared our absorption coefficients with those obtained by squaring the $\gamma \rightarrow e^{+} e^{-}$amplitude directlyt. Our results reduce to that of Daugherty and Harding in the special limit $q^{2}=0$ which they have assumed. This is similar to what Shabad has demonstrated in Ref. [9] as he compared his result with that of Klepikov [4] in the above-mentioned limit for $q^{2}$. Our approach differs from that of Refs. [7,9] in that Shabad performs the calculation in the beyond-threshold energy where the algebraic manipulations are rather involved and precautions are required, whereas we take the advantage of inverse Mellin transform which permits us to calculate the polarization function near the zero energy with a convenient energy expansion. We wish to stress again that our approach is physically intuitive. We have written $\Pi_{\|, \perp}$ as a multiple series in $\omega$ (photon energy), $\alpha$ and $\beta$. It is possible to show that [15], for fixed values of $\alpha$ and $\beta$, the series in $\omega$ begins to diverge at the threshold energy $\omega_{\text {th }} \equiv m_{e}\left(\sqrt{1+2 l_{1} B^{\prime}}+\sqrt{1+2 l_{2} B^{\prime}}\right)$ where the Landau levels $l_{1}$ and $l_{2}$ are given by $l_{1}=\left(\beta+\alpha-1 / B^{\prime}\right) / 2$ and $l_{2}=\left(\beta-\alpha-1 / B^{\prime}\right) / 2$. The divergence of the energy series beginning at $\omega=\omega_{\text {th }}$ implies the existence of absorptive part beyond this point. We are able to compute the absorptive part as well as the dispersive part at any energy with the help of inverse Mellin transform and Kramers-Kronig relation.

In closing, we have developed an integral-transform technique to compute the absorptive part of photon polarization function in a background magnetic field, while the dispersive part can be obtained via the Kramers-Kronig relation. Although we have chosen a special case $\mathbf{q} \cdot \mathbf{B}=0$ for convenience, the result for a general angle between $\mathbf{q}$ and $\mathbf{B}$ is easy to infer. Using the relations $\hat{\Pi}_{\|}\left(\omega^{2}, q_{x}^{2}, \theta\right)=-\left(\alpha_{e} \omega^{2} \sin ^{2} \theta / 4 \pi\right) \bar{\Pi}_{\|}\left(q_{\|}^{2}, q_{x}^{2}\right), \hat{\Pi}_{\perp}\left(\omega^{2}, q_{x}^{2}, \theta\right)=$ $-\left(\alpha_{e} q_{x}^{2} / 4 \pi\right) \bar{\Pi}_{\perp}\left(q_{\|}^{2}, q_{x}^{2}\right)$, and $\hat{\kappa}_{\|, \perp}=\operatorname{Im} \hat{\Pi}_{\|, \perp} / \omega$, it is clear that $\hat{\kappa}_{\|}\left(\omega^{2}, q_{x}^{2}, \theta\right)=-\left(\alpha_{e} \omega \sin ^{2} \theta / 4 \pi\right) \operatorname{Im} \bar{\Pi}_{\|}\left(q_{\|}^{2}, q_{x}^{2}\right)$, and $\hat{\kappa}_{\perp}\left(\omega^{2}, q_{x}^{2}, \theta\right)=-\left(\alpha_{e} q_{x}^{2} / 4 \pi \omega\right) \operatorname{Im}_{\perp}\left(q_{\|}^{2}, q_{x}^{2}\right)$. For $\theta=\pi / 2$, i.e., $\mathbf{q} \cdot \mathbf{B}=0, \bar{\kappa}_{\|} \rightarrow \kappa_{\|}=-\left(\alpha_{e} \omega / 4 \pi\right) \operatorname{Im} \bar{\Pi}_{\|}\left(\omega^{2}, q_{x}^{2}\right)$ and $\bar{\kappa}_{\perp} \rightarrow \kappa_{\perp}=-\left(\alpha_{e} q_{x}^{2} / 4 \pi \omega\right) \operatorname{Im} \bar{\Pi}_{\perp}\left(\omega^{2}, q_{x}^{2}\right)$. Hence, for example, by comparing the above expressions for $\hat{\kappa}_{\|}$and $\kappa_{\|}$, one realizes that $\hat{\kappa}_{\|}$can be inferred from $\kappa_{\|}$by first dividing the latter by $\omega$, then replacing the variable $\omega^{2}$ by $q_{\|}^{2}$, and finally multiplying the entire expression by $\omega \sin ^{2} \theta$. The procedure of obtaining $\hat{\kappa}_{\perp}$ from $\kappa_{\perp}$ is also straightforward. There are other generalizations to the current work. For example, one may analyze the photon polarization function in a general background electromagnetic field, or study other current-current correlation functions under the same external condition. To our knowledge, the vector-axial vector correlation function relevant to weak interaction processes has not been analyzed as detailed as the fashion presented in this work. We shall report the results of such analysis as well as some technical details omitted in this letter in a forthcoming publication [15.

\section{ACKNOWLEDGMENTS}

We thank S.-C. Lee for his comments which lead to this investigation. This work is supported in part by the National Science Council under grant numbers NSC89-2112-M009-043 and NSC89-2112-M009-041.

[1] M. H. Johnson and B. A. Lippmann, Phys. Rev. 76, 828, 1949.

[2] P. A. Sturrock, ApJ 164, 529, 1971.

[3] J. S. Toll, Ph.D. thesis, Princeton Univ., 1952 (unpublished).

[4] N. P. Klepikov, Zh. Eksp. Theor. Fiz., 26, 19, 1954.

[5] J. K. Daugherty and A. K. Harding, ApJ 273, 761, 1983.

[6] W.-y. Tsai and T. Erber, Phys. Rev. D 10, 492, 1974.

[7] A. E. Shabad, Ann. Phys. (N.Y.) 90, 166, 1975.

[8] J. Schwinger, Phys. Rev. 82, 664, 1951.

[9] A. E. Shabad, Lebedev Phys. Inst. preprints NN60, 80, 1974.

[10] W. Dittrich and R. Shaisultanov, Phys. Rev. D 62, 045024, 2000.

[11] C. Schubert, hep-th/0101036.

\footnotetext{
${ }^{4}$ We make comparisons with the most updated results in Ref. 㫬.
} 
[12] W.-y. Tsai, Phys. Rev. D 10, 2699, 1974.

[13] W.-F. Kao, G.-L. Lin and J.-J. Tseng, Phys. Lett. B 495, 105, 2000.

[14] S. L. Adler, Ann. Phys. (N.Y.) 67, 599, 1971.

[15] W.-F. Kao and G.-L. Lin, in preparation. 